\title{
ERRATUM
}

\section{Erratum to: Production setup-sequencing and lot-sizing at an animal nutrition plant through ATSP subtour elimination and patching}

\author{
Alistair R. Clark · Reinaldo Morabito · Eli A.V. Toso
}

Published online: 1 May 2010

(C) Springer Science+Business Media, LLC 2010

Erratum to: J Sched (2010) 13: 111-121

DOI 10.1007/s10951-009-0135-7

$\sum_{i} z_{i t}=1 \quad t=2, \ldots, T+1$

The article was published with misprints in many mathematical expressions. The correct expressions are:

$\sum_{i} y_{i j t} \leq \sum_{k} y_{j k t}+z_{j, t+1} \quad \forall j, t$

$I_{i, t-1}^{+}-I_{i, t-1}^{-}+q_{i t}-d_{i t}=I_{i t}^{+}-I_{i t}^{-} \quad \forall i, t ;$

$p_{i} q_{i t} \leq\left(C_{t}+u_{t}\right) \sum_{j} y_{j i t} \quad \forall t, i \neq i_{0} ;$

$y_{i i t}=0 \quad \forall i, t ;$

$y_{i, i_{0}, t}=0 \quad \forall i, t ;$

$$
\begin{aligned}
& z_{j t}+\sum_{i} y_{i j t} \geq \sum_{k} y_{j k t} \quad \forall j, t ; \\
& z_{j t}+\sum_{i} y_{i j t}=\sum_{k} y_{j k t}+z_{j, t+1} \quad \forall j, t ; \\
& z_{i t} \leq \sum_{j} y_{i j t}+z_{i, t+1} \leq 1 \quad \forall i, t .
\end{aligned}
$$

The online version of the original article can be found under doi:10.1007/s10951-009-0135-7.

\footnotetext{
A.R. Clark $(\bowtie)$

Bristol Institute of Technology, University of the West of England, Frenchay Campus, Bristol BS16 1QY, UK

e-mail: Alistair.Clark@uwe.ac.uk

R. Morabito

Production Engineering Department, Universidade Federal de São Carlos, Campus de São Carlos, Rodovia Washington Luís (SP-310), Km 235, São Carlos, SP 13565-905, Brazil e-mail: Morabito@ufscar.br
}

\section{E.A.V. Toso}

Production Engineering Department, Universidade Federal de São Carlos, Campus de Sorocaba, Rodovia João Leme dos Santos (SP-264), Km 110, Sorocaba, SP 18052-780, Brazil e-mail: Eli@ufscar.br 